\title{
ON ESTIMATING THE INVERSE COEFFICIENTS FOR MEROMORPHIC UNIVALENT FUNCTIONS OMITTING A DISC
}

\author{
HENRYKA SIEJKA and OLLI TAMMI
}

\section{Introduction}

In [2] and [3] the class

$$
\sum_{b}=\left\{H\left|H(z)=z+\sum_{0}^{\infty} A_{n} z^{-n}, \quad\right| z|>1, \quad| H(z) \mid>b \in(0,1)\right\}
$$

of meromorphic univalent functions was defined and considered. This class was found to have a one-to-one relationship with bounded univalent functions

$$
S(b)=\left\{f\left|f(z)=b\left(z+\sum_{2}^{\infty} a_{v} z_{v}\right),\right| z|<1,| f(z) \mid<1, b \in(0,1)\right\}
$$

so that

$$
H(z)=\frac{b}{f\left(\frac{1}{z}\right)}, \quad|z|>1 .
$$

Especially the inverse coefficients, i.e., those of the inverse function $I$ of $H$, were estimated. Denote the inverse function of $w=H(z)$ by

$$
z=I(w)=w+\sum_{0}^{\infty} E_{n} w^{-n} .
$$

In [2] a FitzGerald-Launonen inequality was written for $I$. This appeared to be effective especially for the odd indexes. Thus for odd $\sum_{b}$-functions all the inverse coefficients were maximized. However, for general $\sum_{b}$-functions the FitzGerald-Launonen inequality gave results only for some initial odd inverse coeffcients. Moreover, this method needed the maxima of corresponding initial even inverse coefficients. For estimating the latter the variational method appeared to be the most useful. However, technical difficulties prevented proceeding beyond $\left|E_{4}\right|$ in [3].

In the present paper the general result for inverse $\sum_{b}$-coefficients is derived by applying the variational method, which appears to work equally well for both odd and even indexes. 


\section{The variation of $I$}

The relation (1) allows us to utilize the variational formulae true for $S(b)$ functions. For brevity, adopt directly the notations and results of [4]. Thus (1), p. 137, yields the variational formula of $f \in S(b)$ (here the parameters $A_{v}$ are not to be mixed with the coefficients $A_{v}$ of $H$ ). Through (1) the variational formula for $f$ is transformed into that for $H$ and further for $I$ :

$$
\begin{gathered}
I^{*}(w)=I(w)+I^{\prime}(w) K \cdot \varepsilon+O\left(\varepsilon^{2}\right) ; \\
K=\sum_{1}^{N}\left\{A_{v} \frac{w_{v} w}{w_{v}-w}+\bar{A}_{v} \frac{\bar{w}_{v} w^{2}}{b^{2}-\bar{w}_{v} w}+A_{v}\left(\frac{w_{v} I^{\prime}\left(w_{v}\right)}{I\left(w_{v}\right)}\right)^{2} \frac{I(w)^{2}}{I^{\prime}(w)\left(I(w)-I\left(w_{v}\right)\right)}\right. \\
\left.+\bar{A}_{v}\left(\overline{\frac{w_{v} I^{\prime}\left(w_{v}\right)}{I\left(w_{v}\right)}}\right)^{2} \frac{I(w)}{I^{\prime}(w)\left(I(w) \overline{I\left(w_{v}\right)}-1\right)}\right\} .
\end{gathered}
$$

The expansion form of (2) reads

$$
\begin{gathered}
\sum_{0}^{\infty}\left(E_{n}^{*}-E_{n}\right) w^{-n}=\varepsilon \sum_{1}^{N}\left\{( \frac { w _ { v } I ^ { \prime } ( w _ { v } ) } { I ( w _ { v } ) } ) ^ { 2 } \left[A_{v}\left(w+\left(E_{0}+I\left(w_{v}\right)\right) w^{0}+\ldots\right)\right.\right. \\
\left.\left.+\bar{A}_{v}\left(I\left(w_{v}\right)^{-1} w^{0}+\ldots\right)\right]+A_{v}\left(-w_{v} w^{0}+\ldots\right)+\bar{A}_{v}\left(-w-b^{2} \bar{w}_{v}^{-1} w^{0}+\ldots\right)\right\}+O\left(\varepsilon^{2}\right) .
\end{gathered}
$$

On the right side the coefficient of $w$ appears to be

$$
\sum_{1}^{N}\left\{A_{v}\left(\frac{w_{v} I^{\prime}\left(w_{v}\right)}{I\left(w_{v}\right)}\right)^{2}-\bar{A}_{v}\right\}=0 .
$$

This is due to the fact that according to (4), p. 138, in [4]

$$
b^{*}=b\left[1+\varepsilon \sum_{1}^{N}\left\{A_{v}\left(\frac{w_{v} I^{\prime}\left(w_{v}\right)}{I\left(w_{v}\right)}\right)^{2}-\bar{A}_{v}\right\}\right]+O\left(\varepsilon^{2}\right) .
$$

Hence, for preserving the class $\sum_{b}$, i.e., for keeping $b$ constant in the variation, we must require that the parameters $A_{v}$ are chosen in such a way that (3) holds.

By comparing the coefficients of the above series we obtain the variational formula for the $E_{v}$-coefficients. In order to express this in a useful form we introduce a suitable coefficient-notation, i.e., for any series

$$
W(t)=\sum x_{v} t^{v}
$$

we denote the coefficients by the aid of $W$ :

$$
\varkappa_{v}=[W(t)]_{v} .
$$


Using this we can write

$$
\begin{gathered}
E_{n}^{*}-E_{n}=\varepsilon \sum_{1}^{N}\left(A_{v} X_{v}+\bar{A}_{v} Y_{v}+A_{v} Z_{v}+\bar{A}_{v} U_{v}\right)+O\left(\varepsilon^{2}\right), n=0,1,2, \ldots ; \\
X_{v}=\left(\frac{w_{v} I^{\prime}\left(w_{v}\right)}{I\left(w_{v}\right)}\right)^{2}\left[\frac{I(w)}{\left.1-\frac{I\left(w_{v}\right)}{I(w)}\right]_{-n}},\right. \\
\left.Y_{v}=\left(\frac{\left.\frac{1}{I\left(w_{v} I^{\prime}\left(w_{v}\right)\right.}\right)^{2}}{I\left(w_{v}\right)}\right]_{2} \frac{1}{\overline{I\left(w_{v}\right)} I(w)}\right]_{-n}, \\
Z_{v}=\left[\frac{-w_{v} I^{\prime}(w)}{1-\frac{w_{v}}{w}}\right]_{-n}, \\
U_{v}=\left[\frac{-w I^{\prime}(w)}{b^{2}}\right]_{-n}
\end{gathered}
$$

Applying the notation of [4] we write the variational formula as in (5), p. 138:

$$
\begin{gathered}
E_{n}^{*}=E_{n}+\varepsilon \sum_{1}^{N}\left(A_{v} H_{v}+\bar{A}_{v} K_{v}\right)+O\left(\varepsilon^{2}\right) ; \\
H_{v}=X_{v}+Z_{v}, \quad K_{v}=Y_{v}+U_{v} .
\end{gathered}
$$

(8), p. 140 , corresponds to (3) in the abbreviated form

$$
\sum_{1}^{N}\left(A_{v} e_{v}-\bar{A}_{v}\right)=0 ; \quad e_{v}=\left(\frac{w_{v} I^{\prime}\left(w_{v}\right)}{I\left(w_{v}\right)}\right)^{2} .
$$

For the extremal $E_{n}>0$ the treatment on pp. 140-142 of [4] yields the necessary condition (13), p. 142:

$$
\begin{aligned}
& H_{1}+\bar{K}_{1}+\bar{\lambda} e_{1}-\lambda=0 \\
& \Leftrightarrow \\
& X_{1}+Z_{1}+\bar{Y}_{v}+\bar{U}_{v}+\bar{\lambda} e_{1}-\lambda=0 .
\end{aligned}
$$

Denote $w_{1}=w$. In the expressions ${ }_{v}[$ ] write $w=t$. Thus, the necessary extremal condition assumes the form i.e.,

$$
\left(\frac{w I^{\prime}(w)}{I(w)}\right)^{2}[]+{ }_{3}[]+\left(\frac{w I^{\prime}(w)}{I(w)}\right)^{2}{ }_{2}[]+{ }_{4}[]+\lambda\left(\frac{w I^{\prime}(w)}{I(w)}\right)^{2}-\lambda=0
$$

$$
\begin{gathered}
\left(\frac{w I^{\prime}(w)}{I(w)}\right)^{2}\left\{\left[\frac{I(t)}{1-\frac{I(w)}{I(t)}}\right]_{-n}+\left[\frac{\overline{I(w)^{-1}}}{1-\overline{I(w)^{-1} I(t)^{-1}}}\right]_{-n}+\bar{\lambda}\right\} \\
=-\left[_{3}\left[\frac{-w I^{\prime}(t)}{1-w t^{-1}}\right]_{-n}-\right]_{4}\left[\frac{-t I^{\prime}(t)}{1-b^{2} \bar{w}^{-1} t^{-1}}\right]_{-n}+\lambda .
\end{gathered}
$$


This is the necessary condition for the inverse $\sum_{b}$-function $I$ maximizing $E_{n}>0$. The comparison of coefficients in (4) yields in this case $\lambda \in \mathbf{R}$.

The expansions of the functions in the ${ }_{v}[$ ]-expressions can be written by the aid of

$$
I(t)=t+\sum_{0}^{\infty} E_{v} t^{-v}, \quad I(t)^{-1}=\sum_{1}^{\infty} \alpha_{v} t^{-v} \quad\left(\alpha_{1}=1\right)
$$

as follows $(I(w)=I)$ :

$$
\begin{aligned}
& { }_{1}[]: \frac{I(t)}{1-I \cdot I(t)^{-1}}=t+\left(E_{0}+I\right) t^{0}+\left(E_{1}+I^{2}\right) t^{-1}+\left(E_{2}+\alpha_{2} I^{2}+I^{3}\right) t^{-2} \\
& +\left(E_{3}+\alpha_{2} I^{2}+2 \alpha_{2} I^{3}+I^{4}\right) t^{-3}+\left(E_{4}+\alpha_{4} I^{2}+\left(2 \alpha_{3}+\alpha_{2}^{2}\right) I^{3}+3 \alpha_{2} I^{4}+I^{5}\right) t^{-4}+\ldots, \\
& { }_{2}[]: \frac{\bar{I}^{-1}}{1-\bar{I}^{-1} I(t)^{-1}}=\bar{I}^{-1} \cdot t^{0}+\bar{I}^{-2} t^{-1}+\left(\alpha_{2} \bar{I}^{-2}+\bar{I}^{-3}\right) t^{-2} \\
& +\left(\alpha_{3} \bar{I}^{-2}+2 \alpha_{2} \bar{I}^{-3}+\bar{I}^{-4}\right) t^{-3}+\left(\alpha_{4} \bar{I}^{-2}+\left(2 \alpha_{3}+\alpha_{2}^{2}\right) \bar{I}^{-3}+3 \alpha_{2} \bar{I}^{-4}+\bar{I}^{-5}\right) t^{-4}+\ldots, \\
& { }_{3}[]:-\frac{-w I^{\prime}(t)}{1-w t^{-1}}=w \cdot t^{0}+w^{2} t^{-1}+\left(w^{3}-E_{1} w\right) t^{-2}+\left(w^{4}-E_{1} w^{2}-2 E_{2} w\right) t^{-3} \\
& +\left(w^{5}-E_{1} w^{3}-2 E_{2} w^{2}-3 E_{3} w\right) t^{-4}+\ldots, \\
& { }_{4}[]:-\frac{-t I^{\prime}(t)}{1-b^{2} \bar{w}^{-1} t^{-1}}=t+b^{2} \bar{w}^{-1} \cdot t^{0}+\left(b^{4} \bar{w}^{-2}-E_{1}\right) t^{-1} \\
& +\left(b^{6} \bar{w}^{-3}-E_{1} b^{2} \bar{w}^{-1}-2 E_{2}\right) t^{-2}+\left(b^{8} \bar{w}^{-4}-E_{1} b^{4} \bar{w}^{-2}-2 E_{2} b^{2} \bar{w}^{-1}-3 E_{3}\right) t^{-3} \\
& +\left(b^{10} \bar{w}^{-5}-E_{1} b^{6} \bar{w}^{-3}-2 E_{2} b^{4} \bar{w}^{-2}-3 E_{3} b^{2} \bar{w}^{-1}-4 E_{4}\right) t^{-4}+\ldots
\end{aligned}
$$

As an example consider the case $n=4$.

\section{The coefficient $E_{4}$}

The necessary condition (4) for $E_{4}$ assumes, according to the above developments, the form $(\lambda \in \mathbf{R})$ :

$$
\begin{aligned}
&\left(\frac{w I^{\prime}(w)}{I(w)}\right)^{2}\left(I^{5}+3 \alpha_{2} I^{4}+\left(2 \alpha_{3}+\alpha_{2}^{2}\right) I^{3}+\alpha_{4} I^{2}+\lambda+E_{4}+\bar{\alpha}_{4} I^{-2}+\overline{\left(2 \alpha_{3}+\alpha_{2}^{2}\right)} I^{-3}\right. \\
&\left.+3 \bar{\alpha}_{2} I^{-4}+I^{-5}\right)=w^{5}-E_{1} w^{3}-2 E_{2} w^{2}-3 E_{3} w+\lambda-4 E_{4}-3 \bar{E}_{3} b^{2} w^{-1} \\
&-2 \bar{E}_{2} b^{4} w^{-2}-\bar{E}_{1} b^{6} w^{-3}+b^{10} w^{-4} .
\end{aligned}
$$

In [1] Netanyahu maximizes the $E_{v}$-coefficient for $\sum$ i.e., in the special case $b=0$ by the aid of the variational formula. In agreement with him denote

$$
E_{v}=-\beta_{v} \quad(v=0,1, \ldots)
$$

and maximize the $\beta_{v}$-coefficients. Because

$$
z=I(w)=w-\sum_{0}^{\infty} \beta_{n} w^{-n}
$$


and thus

$$
\frac{1}{I(w)}=\Sigma_{1}^{\infty} \alpha_{v} w^{-v}=\frac{1}{w\left(1-\sum_{0}^{\infty} \beta_{v} w^{-v-1}\right)},
$$

we see that $\alpha_{v}$ is a polynomial $P_{v}\left(\beta_{0}, \ldots, \beta_{v-2}\right)$ with only positive coefficients. For example,

$$
\begin{aligned}
& \alpha_{2}=\beta_{0}, \\
& \alpha_{3}=\beta_{0}^{2}+\beta_{1}, \\
& \alpha_{4}=2 \beta_{0} \beta_{1}+\beta_{0}^{3}+\beta_{2} .
\end{aligned}
$$

This remark appears to be useful when estimating coefficients of the differential equation.

Rewrite (5) for the extremal $H(z)$ by using the $\alpha_{v}$ - and $\beta_{v}$-coefficients. Remember that $E_{4}>0$ so that $\beta_{4}=-E_{4}<0$.

$$
\left(\frac{z H^{\prime}}{H}\right)^{2} \times
$$

$$
\begin{gathered}
\times\left(H^{5}+\beta_{1} H^{3}+2 \beta_{2} H^{2}+3 \beta_{3} H+\lambda+4 \beta_{4}+3 \bar{\beta}_{3} b^{2} H^{-1}+2 \bar{\beta}_{2} b^{4} H^{-2}+\bar{\beta}_{1} b^{6} H^{-3}+b^{10} H^{-5}\right)^{1} \\
=\left(z^{5}+v_{42} z^{4}+v_{43} z^{3}+v_{44} z^{2}+\lambda-\beta_{4}+\bar{v}_{44} z^{-2}+\bar{v}_{43} z^{-3}+\bar{v}_{42} z^{-4}+z^{-5}\right)_{2} ; \\
v_{42}=3 \alpha_{2}=3 \beta_{0}, \\
v_{43}=2 \alpha_{3}+\alpha_{2}^{2}=3 \beta_{0}^{2}+2 \beta_{1}, \\
v_{44}=\alpha_{4}=2 \beta_{0} \beta_{1}+\beta_{0}^{3}+\beta_{2} .
\end{gathered}
$$

In [3] all the lower coefficients included are maximized essentially by the radialslit mapping $I_{r}$ which satisfies the condition

$$
\begin{gathered}
I_{r}+I_{r}^{-1}=w-2(1-b)+b^{2} w^{-1} \\
I_{r}(w)=\frac{w+b^{2} w^{-1}+2 b-2}{2}+\frac{w}{2} \times
\end{gathered}
$$

$$
\begin{gathered}
\times\left[1+4(b-1) w^{-1}+\left(6 b^{2}-8 b\right) w^{-2}+4 b^{2}(b-1) w^{-3}+b^{4} w^{-4}\right]^{1 / 2}=w+\sum_{0}^{\infty}\left(-B_{v}\right) w^{-v} ; \\
B_{0}=2(1-b), \quad B_{1}=1-b^{2}, \\
B_{v}=\gamma_{n 0}+\gamma_{n 1} b+\gamma_{n 2} b^{2}+\ldots+\gamma_{n(n-1)} b^{n-1} ; \\
\gamma_{n v}=(-1)^{v} \frac{2}{v !} \frac{n-v}{n-v+1} \frac{(2 n-v-1) !}{[(n-v) !]^{2}} .
\end{gathered}
$$

The numbers $B_{v}$ are thus the maxima of $\beta_{v}$. Assume here that the inequalities

$$
\left|\beta_{v}\right| \leqq B_{v} \quad(v=0,1,2,3)
$$

are true and maximize $\beta_{4}$ by using the necessary condition (6). 
As in [3], use the fact that the extremal image-domain has at least one slit, i.e., ()$_{1}=0$ at the starting point $b e^{i \psi}$ of the slit and $\lambda+4 \beta_{4}$ is determined by ()$_{1} \geqq 0$, $\psi \in[0,2 \pi]$, that is,

$$
\begin{aligned}
\lambda+4 \beta_{4} & =\max \left[-2 \operatorname{Re}\left\{b^{5} e^{i 5 \psi}+\beta_{1} b^{3} e^{i 3 \psi}+2 \beta_{2} b^{2} e^{i 2 \psi}+3 \beta_{3} b e^{i \psi}\right\}\right] \\
& =\max \left[2 \operatorname{Re}\left\{-b^{5} e^{i 5 \psi}+E_{1} b^{3} e^{i 3 \psi}+2 E_{2} b^{2} e^{i 2 \psi}+3 E_{3} b e^{i \psi}\right\}\right] \\
& \leqq 2\left(b^{5}+\left|\beta_{1}\right| b^{3}+2\left|\beta_{2}\right| b^{2}+3 b\left|\beta_{3}\right|\right) \\
& \leqq 2\left(b^{5}+b^{3} B_{1}+2 b^{2} B_{2}+3 b B_{3}\right) \\
& =2\left(15 b-20 b^{2}+6 b^{3}\right) .
\end{aligned}
$$

Equality here holds for $\psi=0$ and $I=I_{r}$, which has $E_{v}=-B_{v}<0$, when properly rotated. Thus

$$
\lambda+4 \beta_{4}=2\left(15 b-20 b^{2}+6 b^{3}\right) .
$$

( ) $)_{2}$ has at least one zero $e^{i \varphi}$, the pre-image of the endpoint of the slit. Thus

$$
\begin{gathered}
\beta_{4}-\lambda=-5\left|\beta_{4}\right|-\left(30 b-40 b^{2}+12 b^{3}\right)=-2 \operatorname{Re}\left\{e^{i 5 \varphi}+v_{42} e^{i 4 \varphi}+v_{43} e^{i 3 \varphi}+v_{44} e^{i 2 \varphi}\right\} ; \\
5\left|\beta_{4}\right| \leqq-\left(30 b-40 b^{2}+12 b^{3}\right)+2\left(1+\left|v_{42}\right|+\left|v_{43}\right|+\left|v_{44}\right|\right) .
\end{gathered}
$$

The numbers $\left|v_{n v}\right|$ are maximized with $\left|\beta_{v}\right|$ :

$$
\begin{aligned}
\left|v_{42}\right| & \leqq 6(1-b), \\
\left|v_{43}\right| & \leqq 14-24 b+10 b^{2}, \\
\left|v_{44}\right| & \leqq 14-30 b+20 b^{2}-4 b^{3} ; \\
\left|\beta_{4}\right| & \leqq \frac{1}{5}\left[-\left(30 b-40 b^{2}+12 b^{3}\right)+70-120 b+60 b^{2}-8 b^{3}\right] \\
& =14-30 b+20 b^{2}-4 b^{3} .
\end{aligned}
$$

Again, the equality belongs essentially to $I_{r}$, because this holds for all the lower coefficients used in the triangle inequality estimations. - This result is the one proved in the above manner in [3].

\section{The general case}

From (4) we obtain for $E_{n}=-\beta_{n}>0$ the necessary condition for the extremal $H$ :

$$
\begin{gathered}
\left(\frac{z H^{\prime}}{H}\right)^{2} \times \\
\times\left(H^{n+1}+\sum_{1}^{n-1} v \beta_{v} H^{n-v}+\lambda+n \beta_{n}+\sum_{n-1}^{1} v \bar{\beta}_{v} b^{2 n-2 v} H^{-n+v}+b^{2 n+2} H^{-n-1}\right)_{1} \\
=\left(z^{n+1}+\sum_{2}^{n} v_{n v} z^{n+2-v}+\lambda-\beta_{n}+\sum_{n}^{2} \bar{v}_{n v} z^{-n-2+v}+z^{-n-1}\right)_{2} .
\end{gathered}
$$

The numbers $v_{n v}$ are polynomials $Q_{v}\left(\beta_{0}, \ldots, \beta_{v-2}\right)$ with only positive coefficients. 
Assume that the lower coefficients $\beta_{0}, \ldots, \beta_{n-1}$ are all maximized by $I_{r}$ defined by (7). We proceed in maximizing $\left|\beta_{n}\right|$ by imitating the procedure of Section 3 . Thus, ()$_{1}$ has at least one zero $b e^{i \psi}$ which determines $\lambda+n \beta_{n}$ so that ()$_{1} \geqq 0$ for $\psi \in[0,2 \pi]$ :

$$
\begin{aligned}
& \lambda+n \beta_{n}=\max \left[-2 \operatorname{Re}\left\{b^{n+1} e^{i(n+1) \psi}+\sum_{1}^{n-1} v \beta_{v} b^{n-v} e^{i(n-v) \psi}\right\}\right] \\
&=\max \left[2 \operatorname{Re}\left\{-b^{n+1} e^{i(n+1) \psi}+\sum_{1}^{n-1} v E_{v} b^{n-v} e^{i(n-v) \psi}\right\}\right] \\
& \leqq 2\left(b^{n+1}+\sum_{1}^{n-1} v\left|\beta_{v}\right| b^{n-v}\right) \\
& \leqq 2\left(b^{n+1}+\sum_{1}^{n-1} v B_{v} b^{n-v}\right) \\
&=2 \sum_{1}^{n-1} C_{v} b^{v} ; \\
& C_{v}=\frac{(-1)^{v-1}}{(v-1) !} \frac{n-v}{n-v+1} \frac{(2 n-v-1) !}{[(n-v) !]^{2}}=-\frac{v}{2} \gamma_{n v} \quad(v=1,2, \ldots, n-1) .
\end{aligned}
$$

Again, equality holds for $\psi=0$ and for a rotated $I_{r}$; hence

$$
\lambda+n \beta_{n}=2 \sum_{1}^{n-1} C_{v} b^{v} .
$$

()$_{2}=0$ at some $e^{i \varphi}$ and thus

$$
\begin{aligned}
\beta_{n}-\lambda & =-(n+1)\left|\beta_{n}\right|-2 \sum_{1}^{n-1} C_{v} b^{v} \\
& =2 \operatorname{Re}\left\{e^{i(n+1) \varphi}+\sum_{2}^{n} v_{n v} e^{i(n+2-v) \varphi}\right\} ; \\
(n+1)\left|\beta_{n}\right| & \leqq-2 \sum_{1}^{n-1} C_{v} b^{v}+2\left(1+\sum_{2}^{n}\left|v_{n v}\right|\right)_{0} .
\end{aligned}
$$

In order to estimate ( $)_{0}$ we return to the general form (4) of the differential equation. The coefficients $v_{n v}$ are defined by ${ }_{1}[$ ] , i.e., they are those of

$$
\frac{I(t)}{1-I \cdot I(t)^{-1}}=I(t)+I+I^{2} \cdot I(t)^{-1}+I^{3} \cdot I(t)^{-2}+\ldots
$$

We are actually interested in the non-constant term of the $I$-polynomials (cf. the expansion of $\left.{ }_{1}[]\right)$. Because the constant term $E_{v}$ is due to the first term $I(t)$, we may confine ourselves to the expansion

$$
\begin{aligned}
F(t)= & \frac{I(t)}{1-I \cdot I(t)^{-1}}-I(t)=\frac{I}{1-I \cdot I(t)^{-1}} \\
= & I \cdot t^{0}+I^{2} \cdot t^{-1}+\left(\alpha_{2} I^{2}+I^{3}\right) t^{-2}+\left(\alpha_{3} I^{2}+2 \alpha_{2} I^{3}+I^{4}\right) t^{-3} \\
& +\left(\alpha_{4} I^{2}+\left(2 \alpha_{3}+\alpha_{2}^{2}\right) I^{3}+3 \alpha_{2} I^{4}+I^{5}\right) t^{-4}+\ldots \\
= & I \cdot t^{0}+I^{2} \cdot t^{-1}+\sum_{n=2}^{\infty}\left(\sum_{v=2}^{n} v_{n(n-v+2)} I^{v}+I^{n+1}\right) t^{-n} .
\end{aligned}
$$

Because the numbers $v_{n v}$ are polynomials of $\beta_{0}, \ldots, \beta_{v-2}$ with only positive coefficients, we obtain their maxima $V_{n v}$ by writing $\beta_{k}=B_{k}$ in those polynomials. Actually, 
we need only the maximal sum $u_{n}=1+\sum_{v=2}^{n} V_{n v}$, which is thus obtained as the coefficient $u_{v}$ of $F(t)$ with $I=1$ and $I_{r}(t)$ defined by $(7)(w=t)$. Thus we have the expansion

$$
\begin{gathered}
I_{r}(t)=t-B_{0}-B_{1} t^{-1}-B_{2} t^{-2}-\ldots \\
\frac{I_{r}(t)}{I_{r}(t)-1}=1+t^{-1}+u_{2} t^{-2}+u_{3} t^{-3}+\ldots ; \\
u_{n}=1+\sum_{v=2}^{n} V_{n v} .
\end{gathered}
$$

The multiplication of the $u_{v}$-expansion by

$$
I_{r}(t)-1=t-\left(1+B_{0}\right)-B_{1} t^{-1}-B_{2} t^{-2}-\ldots
$$

yields the recursion formula:

$$
\begin{aligned}
& u_{2}=1+B_{0}, \\
& u_{3}=\left(1+B_{0}\right) u_{2}+B_{1}, \\
& \ldots \\
& u_{n}=\left(1+B_{0}\right) u_{n-1}+\sum_{v=2}^{n-1} B_{v-1} u_{n-v} \quad\left(u_{1}=1 ; n=2,3, \ldots\right) .
\end{aligned}
$$

This gives

$$
u_{n}=\sum_{v=0}^{n-1} \frac{(-1)^{v}}{v !}(n-v) \frac{(2 n-v-1) !}{[(n-v) !]^{2}} b^{v}=\sum_{v=0}^{n-1} \frac{n-v+1}{2} \gamma_{n v} b^{v} .
$$

According to (9) we now obtain

$$
\begin{gathered}
(n+1)\left|\beta_{n}\right| \leqq \\
2 \sum_{1}^{n-1} \frac{v}{2} \gamma_{n v} b^{v}+2 \sum_{0}^{n-1} \frac{n-v+1}{2} \gamma_{n v} b^{v} \\
=(n+1) \sum_{0}^{n-1} \gamma_{n v} b^{v} \\
\quad\left|\beta_{n}\right| \leqq \sum_{0}^{n-1} \gamma_{n v} b^{v}=B_{n} .
\end{gathered}
$$

Because the lower coefficients $\beta_{0}, \ldots, \beta_{n-1}$ are all maximized, save the rotation, by $I_{r}$, the same holds in (11) and thus for all $n$. Hence we have generalized the result of Netanyahu [1].

Theorem. For the inverse $\sum_{b}$-functions

$$
I(w)=w-\sum_{0}^{\infty} \beta_{v} w^{-v}, \quad|w|>1,
$$

the coefficient estimation

holds.

$$
\begin{gathered}
\left|\beta_{0}\right| \leqq 2(1-b), \quad\left|\beta_{1}\right| \leqq 1-b^{2}, \quad\left|\beta_{n}\right| \leqq \sum_{0}^{n-1} \gamma_{n v} b^{v} \quad(n=2,3, \ldots) ; \\
\gamma_{n v}=(-1)^{v} \frac{2}{v !} \frac{n-v}{n-v+1} \frac{(2 n-v-1) !}{[(n-v) !]^{2}} \quad(v=0, \ldots, n-1)
\end{gathered}
$$


Essentially the only maximizing function is the radial-slit mapping $I_{r}$ defined by

$$
I_{r}+I_{r}^{-1}=w-2(1-b)+b^{2} w^{-1} .
$$

The coefficient $\beta_{1}$ has a one-parametric family of maximizing functions I from

$$
I+I^{-1}=w-c+b^{2} w^{-1}
$$

with $c \in[-2(1-b), 2(1-b)]$ as a parameter (cf. [3]).

This result generalizes the classical theorem of Netanyahu [1] showing that also in the cases other than $b=0$ the radial-slit mapping preserves its extremal role with respect to the inverse coefficients.

\section{References}

[1] Netanyahu, E.: Extremal problems for schlicht functions in the exterior of the unit circle. Canad. J. Math. 17, 1965, 335-341.

[2] SIEJKA, H.: On meromorphic univalent functions omitting a disc. - Israel J. Math. 54, 3, 1986, 291-299.

[3] SIEJKA, H.: On estimating some initial inverse coefficients for meromorphic univalent functions omitting a disc. - To appear in Math. Scand.

[4] TAMm, O.: Extremum problems for bounded univalent functions. - Lecture Notes in Mathematics 646. Springer-Verlag, Berlin-Heidelberg-New York, 1978.

Łódź University

Institute of Mathematics

90-238 Łódź

Poland
University of Helsinki

Department of Mathematics

SF-00100 Helsinki

Finland

Received 25 February 1986 